Article

\title{
Function-Based Tractography of the Language Network Correlates with Aphasia in Patients with Language-Eloquent Glioblastoma
}

\author{
Haosu Zhang ${ }^{1}$, Severin Schramm ${ }^{1}$, Axel Schröder ${ }^{1}$, Claus Zimmer $^{2}$, Bernhard Meyer ${ }^{1}$, \\ Sandro M. Krieg ${ }^{1,3(1)}$ and Nico Sollmann ${ }^{2,3, *(1)}$ \\ 1 Department of Neurosurgery, Klinikum rechts der Isar, Technische Universität München, Ismaninger Str. 22, \\ 81675 Munich, Germany; Haosu.Zhang@gmail.com (H.Z.); Schrammseverin@gmail.com (S.S.); \\ Axel.Schroeder@tum.de (A.S.); Bernhard.Meyer@tum.de (B.M.); Sandro.Krieg@tum.de (S.M.K.) \\ 2 Department of Diagnostic and Interventional Neuroradiology, Klinikum rechts der Isar, \\ Technische Universität München, Ismaninger Str. 22, 81675 Munich, Germany; Claus.Zimmer@tum.de \\ 3 TUM-Neuroimaging Center, Klinikum rechts der Isar, Technische Universität München, \\ 81675 Munich, Germany \\ * Correspondence: nico.sollmann@tum.de
}

Received: 5 June 2020; Accepted: 23 June 2020; Published: 1 July 2020

check for updates

\begin{abstract}
To date, the structural characteristics that distinguish language-involved from non-involved cortical areas are largely unclear. Particularly in patients suffering from language-eloquent brain tumors, reliable mapping of the cortico-subcortical language network is of high clinical importance to prepare and guide safe tumor resection. To investigate differences in structural characteristics between language-positive and language-negative areas, 20 patients (mean age: $63.2 \pm 12.9$ years, 16 males) diagnosed with language-eloquent left-hemispheric glioblastoma multiforme (GBM) underwent preoperative language mapping by navigated transcranial magnetic stimulation (nTMS) and nTMS-based diffusion tensor imaging fiber tracking (DTI FT). The number of language-positive and language-negative points as well as the gray matter intensity (GMI), normalized volumes of U-fibers, interhemispheric fibers, and fibers projecting to the cerebellum were assessed and compared between language-positive and language-negative nTMS mappings and set in correlation with aphasia grades. We found significantly lower GMI for language-positive nTMS points (5.7 \pm 1.7 versus $7.1 \pm 1.6, p=0.0121$ ). Furthermore, language-positive nTMS points were characterized by an enhanced connectivity profile, i.e., these points showed a significantly higher ratio in volumes for U-fibers $(p \leq 0.0056)$, interhemispheric fibers $(p=0.0494)$, and fibers projecting to the cerebellum ( $p=0.0094)$. The number of language-positive nTMS points $(\mathrm{R} \geq 0.4854, p \leq 0.0300)$ as well as the ratio in volumes for $\mathrm{U}$-fibers $(\mathrm{R} \leq-0.4899, p \leq 0.0283)$ were significantly associated with aphasia grades, as assessed pre- or postoperatively and during follow-up examinations. In conclusion, this study provides evidence for structural differences on cortical and subcortical levels between language-positive and language-negative areas, as detected by nTMS language mapping. The results may further increase confidence in the technique of nTMS language mapping and nTMS-based tractography in the direct clinical setting. Future studies may confirm our results in larger cohorts and may expand the findings to patients with other tumor entities than GBM.
\end{abstract}

Keywords: brain stimulation; fiber tractography; glioblastoma multiforme; gray matter; language mapping; navigated transcranial magnetic stimulation 


\section{Introduction}

Resection of intracranial glioma aims at a maximum extent of resection, which should ideally be achieved without causing surgery-related functional deficits that could severely reduce the patients' quality of life [1-4]. To establish maximum resection whilst avoiding functional decline as far as possible, several pre- and intraoperative techniques have been developed to assist in neurosurgical planning and resection guidance [4-7]. For the intraoperative setting, cortical and subcortical direct electrical stimulation (DES) serves as the current gold-standard method [8-10]. Regarding the preoperative setting, navigated transcranial magnetic stimulation (nTMS) has found its way into neurosurgery over the last decade $[6,11,12]$.

The technique of nTMS has lately been used to conduct language mappings in patients suffering from language-eloquent glioma or other entities of brain tumors [13-16]. Furthermore, it has been combined with diffusion tensor imaging (DTI) derived from preoperative magnetic resonance imaging (MRI) to provide spatially resolved maps that visualize language-related structures [17-25]. Integration into clinical routine and the perioperative workflow is seamless, and the approach is currently regarded as a valuable adjunct to intraoperative DES [14,15]. However, in direct comparison to intraoperative DES, nTMS language mapping has shown a rather low specificity of $23.8 \%$ and a positive predictive value of $35.6 \%$ [13]. The mere additional use of nTMS-based DTI fiber tracking (DTI FT) did not improve the identification of DES-positive language areas during awake surgery [24]. Nevertheless, the visualization of the subcortical language network becomes possible purely based on functional data by using nTMS-based DTI FT, which has shown high potential for surgical planning, resection guidance, and risk assessment in patients with language-eloquent lesions [17-25]. However, explorations of the differences between language-positive and language-negative nTMS mappings are still largely missing, which is one reason contributing to the lack of understanding of the comparatively low specificity of nTMS language mapping in relation to intraoperative DES. Further insights may lead to a better definition of the role of nTMS in the neurosurgical setting and to improved understanding of nTMS characteristics.

The cortico-subcortical network behind human language function is complex [26-29]. On the cortical level, differences in gray matter (GM) distribution could probably differentiate between language-related and non-related areas or, at least, between highly and less involved areas. In this regard, previous research has demonstrated that the degree to which language is lateralized to one of the hemispheres is positively predicted by the degree to which GM is lateralized on a voxel-by-voxel basis [30]. In subjects with dyslexia, a GM deficit involving a fronto-temporal network important for phonological processing was revealed, whereas region-specific increases in GM volume are possible for developmental language disorders as a result of compensatory mechanisms [31,32]. On the subcortical level, differences in connectivity profiles between areas depending on language involvement seem likely. Besides major language-related white matter tracts known to be involved in language processing, various short or long interconnecting fibers, such as short association fibers (commonly referred to as arcuate fibers or U-fibers), interhemispheric transcallosal fibers, and fibers projecting to the cerebellum play a role $[28,29,33,34]$.

Against this background, this study's objective is to systematically explore characteristics of language-positive structures according to nTMS language mapping and nTMS-based DTI FT in direct comparison to language-negative counterparts. We hypothesize that (1) language-positive cortical areas may show different focal GM intensity (GMI, as a potential expression of higher GM density as a correlate of increased functional involvement), and that (2) language-positive cortical areas show a different connectivity profile (higher volumes of U-fibers, transcallosal fibers, and fibers projecting to the cerebellum) when compared to language-negative cortical areas. 


\section{Materials and Methods}

\subsection{Ethics}

The current study is in accordance with the Declaration of Helsinki and its later amendments and has been approved by the local institutional review board (registration numbers: 2793/10, 5811/13, 223/14, and 336/17). Written informed consent was obtained from all patients enrolled in this study.

\subsection{Patients and Study Inclusion}

This study is a post-hoc analysis including patients of our prospectively enrolled cohort that underwent language mapping by nTMS and nTMS-based DTI FT of language-related fiber tracts prior to resection of a brain tumor. The following inclusion criteria were defined for the present study:

(1) Written informed consent,

(2) Age above 18 years,

(3) German as first language,

(4) Left-hemispheric perisylvian tumor location (MRI suggesting infiltration and/or compression of anatomically suspected cortical language-eloquent areas and/or suspected close proximity to subcortical language-related pathways),

(5) Availability of preoperative 3-Tesla MRI, including a DTI sequence with 32 diffusion directions,

(6) Clinical indication for preoperative nTMS language mapping and nTMS-based DTI FT,

(7) Surgery for tumor resection and final diagnosis of a glioblastoma multiforme (GBM) according to histopathological examination (based on tumor tissue probes taken during resection), and

(8) Follow-up time of at least 3 months after surgery.

The exclusion criteria were defined as follows:

(1) Multilingual background (regular input in more than one language between birth and adolescence),

(2) Neurological or psychiatric diseases (except for the diagnosis of a GBM), and

(3) Aphasia to a degree not allowing for preoperative language mapping by nTMS.

\subsection{Clinical Examination}

A standardized assessment of sensory function, coordination, muscle strength, and cranial nerve function was performed as part of the initial clinical examination. In particular, the language status was evaluated by a neuropsychologist using the Aachen Aphasia Test and by categorizing language deficits on a four-point scale [14,35-37]. In detail, four grades were established, which were no deficit (grade 0 ), mild deficit (grade 1: normal language comprehension and/or conversational language with slight amnesic aphasia, adequate communication ability), medium deficit (grade 2: minor disruption of language comprehension and/or conversational language, adequate communication ability), and severe deficit (grade 3: major disruption of language comprehension and/or conversational language, clear impairment of communication ability) [14,35-37]. Furthermore, handedness was assessed by the Edinburgh Handedness Inventory (EHI) [38]. The clinical examinations including detailed assessments of language function were repeated postoperatively and during the routine follow-up examinations.

\subsection{Cranial Magnetic Resonance Imaging}

Imaging was performed on a 3-Tesla MRI scanner using a 32-channel head coil (Achieva dStream or Ingenia; Philips Healthcare, Best, The Netherlands). As part of a standardized, multi-sequence imaging protocol for brain tumors, a three-dimensional fluid attenuated inversion recovery (FLAIR) sequence (repetition time (TR)/echo time (TE): $4800 / 277 \mathrm{~ms}, 1 \mathrm{~mm}^{3}$ isovoxel covering the whole head), three-dimensional T1-weighted gradient echo sequence (TR/TE: 9/4 ms, $1 \mathrm{~mm}^{3}$ isovoxel covering the 
whole head) without and with application of an intravenous contrast agent (Dotagraf, Jenapharm GmbH \& Co. KG, Jena, Germany), and a DTI sequence (TR/TE 5000/78 ms, voxel size of $2 \times 2 \times 2 \mathrm{~mm}^{3}$, 32 diffusion gradient directions) were acquired preoperatively and were used for nTMS language mapping (contrast-enhanced T1-weighted sequences) and nTMS-based DTI FT (contrast-enhanced T1-weighted, DTI, and FLAIR sequences).

Postoperative (within the first $48 \mathrm{~h}$ after surgery) and follow-up imaging were performed using the same imaging protocol. In postoperative MRI, special attention was paid to the assessment of residual tumor tissue or achieved gross total resection (GTR) as well as perioperative bleeding or ischemia.

\subsection{Language Mapping by Navigated Transcranial Magnetic Stimulation}

\subsubsection{Mapping Procedure}

Preoperative language mapping by nTMS of the tumor-affected left hemisphere (LH) was carried out during the days before scheduled surgery for tumor resection using a Nexstim eXimia NBS system (version 4.3; Nexstim Plc, Helsinki, Finland) [11,14,15,39,40]. Language mappings were conducted in German as the native language of all enrolled patients.

After initial co-registration of the patient's head and the contrast-enhanced T1-weighted image dataset, pictures of common objects were presented to the patient in the context of two baseline trials (presentation of objects without simultaneous stimulation) [13,40-44]. The objects shown were part of a standardized object-naming task, and the purpose of baseline assessment was to systematically discard objects that did not elicit a quick and fluent response and to familiarize the patients with the task and setup. The remaining stack of objects that were named correctly and fluently was then shown under stimulation of up to 46 target points that had been placed on the LH, with each point being stimulated six times in total (stimulation intensity: $100 \%$ of the individual resting motor threshold, stimulation frequency: $5 \mathrm{~Hz} / 5$ pulses) [13,40,42,43].

The 46 target points for stimulation were tagged with close respect to the cortical parcellation system (CPS), thus covering almost the whole LH except for polar, occipital, and inferior temporal regions, similar to previous studies on nTMS language mapping (Figure 1) [13,42,43]. Exclusion was due to considerable muscle activation and/or discomfort that can be observed following stimulation of these particular regions. Furthermore, in patients showing large tumor masses, the number of 46 target points for stimulation needed to be reduced according to individual cortical architecture given destructions and derangements caused by such lesions. The picture-to-trigger interval during stimulation was set as $0 \mathrm{~ms}$ by default $[45,46]$. The patients' performance during object naming was video-recorded for later evaluation to detect and categorize errors elicited by targeted nTMS [41,44].

\subsubsection{Mapping Evaluation}

Video data of the nTMS language mappings were systematically searched for naming errors of different categories after the mapping procedure. No responses, performance errors, hesitations, neologisms, phonological paraphasias, and semantic paraphasias were defined and considered during evaluation [13,41-44]. The stimulation points that elicited any naming error except hesitations (no responses, performance errors, neologisms, phonological paraphasias, and semantic paraphasias together) were considered as language-positive nTMS points. Correspondingly, stimulation points that did not elicit a naming error were defined as language-negative nTMS points.

Per patient, two export datasets were then generated, which included the language-positive nTMS points of the LH and the language-negative nTMS points of the LH, respectively. Each export file was in Digital Imaging and Communications in Medicine (DICOM) format and included the nose and ears as anatomical landmarks together with the stimulation points. Furthermore, in each patient, CPS regions were counted as language-positive CPS regions when they contained at least one language-positive nTMS point, while CPS regions without language-positive nTMS points were considered as language-negative CPS regions. CPS regions that were not stimulated (polar, occipital, 
and inferior temporal regions) were not considered. The total number of language-positive CPS regions was recorded as $N_{p}$, and the number of language-negative CPS regions as $N_{n}$.

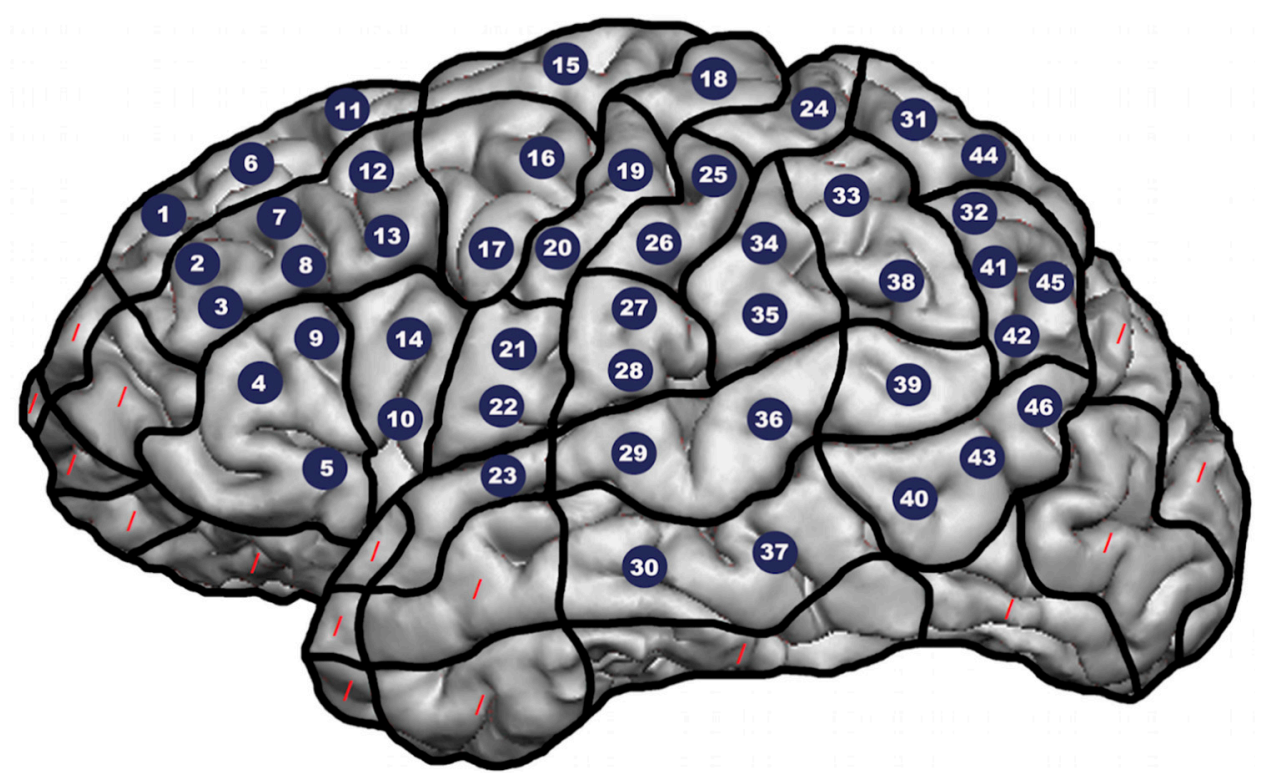

Figure 1. Cortical parcellation system (CPS). The tumor-affected left hemisphere (LH) was mapped by navigated transcranial magnetic stimulation (nTMS) with respect to 46 target points that were placed in relation to the CPS (reduced numbers of target points were accepted in patients with large tumor masses, which hampered placement of the total amount of 46 target points). The points were stimulated six times in total each, with a stimulation intensity of $100 \%$ of the individual resting motor threshold and a stimulation frequency of $5 \mathrm{~Hz} / 5$ pulses. Numbers in circles schematically represent the stimulation targets on a standardized brain template, red dashes mark regions that were not subject to nTMS.

\subsection{Tractography Based on Navigated Transcranial Magnetic Stimulation}

Deterministic fiber tractography was based on nTMS language mapping data without additional anatomical seeding (Brainlab Elements, version 3.1.0; Brainlab AG, Munich, Germany). Both export datasets were first transferred to an external server and then auto-fused with the MRI sequences of the respective patient, using manual correction in case of registration misalignments and eddy current correction for DTI data. The datasets containing language-positive or language-negative nTMS points were separately defined as three-dimensional objects, followed by generation of regions of interest (ROIs) out of these objects by adding a rim of $5 \mathrm{~mm}$ to each stimulation point [14,15,17-19].

An individual fractional anisotropy (FA) value, the fractional anisotropy threshold (FAT), was defined separately in each patient for nTMS-based DTI FT using the ROI constituted of language-positive or language-negative nTMS points, which was determined by setting angulation to $90^{\circ}$, the minimum fiber length (FL) to $30 \mathrm{~mm}$, and increasing the FA stepwise until no fibers were displayed, followed by decreasing the FA by 0.01 , thus visualizing a minimum fiber course. The corresponding FA value was defined as 100\% FAT. A similar approach using a higher minimum FL for FAT determination has been used previously for nTMS-based DTI FT [17,19,47].

After FAT definition, nTMS-based DTI FT was carried out separately with the ROIs of language-positive and language-negative nTMS points using the respective FA values for $100 \%$ FAT, 75\% FAT, 50\% FAT, and 25\% FAT, and a minimum FL of $30 \mathrm{~mm}$ as well as $3 \mathrm{~mm}$, respectively. The values of 30 and $3 \mathrm{~mm}$ were considered for the minimum FL due to the characteristic length of U-fibers that have been described to be typically in this range [48]. 


\subsection{Data Analyses}

The data analyses were divided into two parts (Figure 2). The first part focused on the cortical level to investigate differences in GMI between language-positive and language-negative nTMS points (Figure 2). The second part focused on the subcortical level to assess differences in fiber tractography between nTMS-based DTI FT using language-positive or language-negative nTMS points as ROIs by means of investigating short fibers for short-distance connections (U-fibers), long fibers projecting to the contralateral hemisphere (Cross-F), and fibers projecting to the cerebellum (Cereb-F; Figure 2).

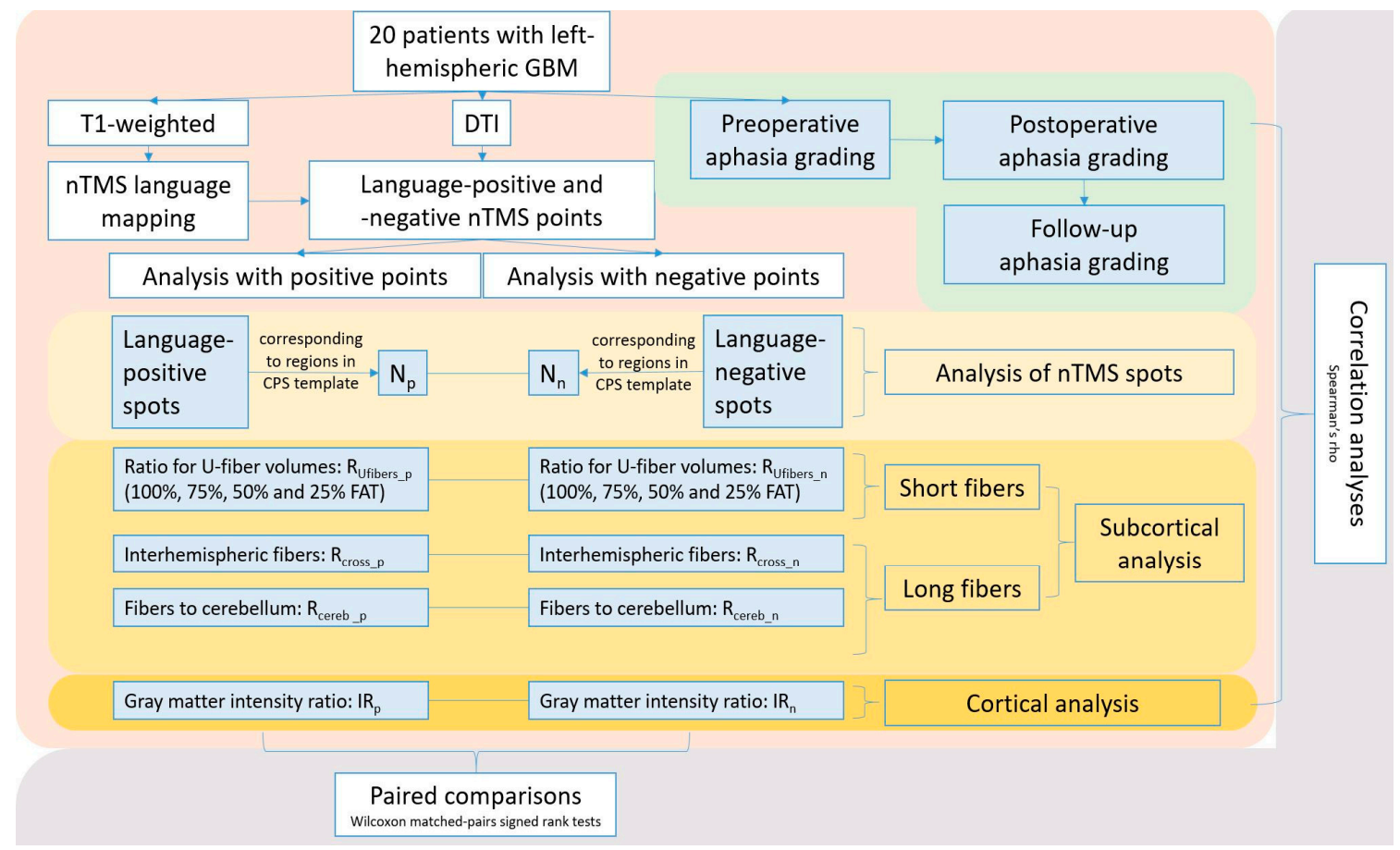

Figure 2. Overview of procedures and analyses. This scheme summarizes the study's steps of analyses, which were performed based on data derived from language mapping by navigated transcranial magnetic stimulation (nTMS) and nTMS-based diffusion tensor imaging fiber tracking (DTI FT). Language-positive and language-negative nTMS points were used separately for the different analyses. The study cohort included 20 patients with left-hemispheric glioblastoma multiforme (GBM).

\subsubsection{Gray Matter Intensity}

Using the T1-weighted sequences co-registered to language-positive or language-negative nTMS points, the GMI was measured per stimulation point (iPlan Net server, version 3.0.1; Brainlab AG, Munich, Germany). Three pixels were randomly selected within a stimulation point and used for GMI measurements to represent the GMI per stimulation point by averaging the three values obtained (Figure 3). The mean GMI of language-positive nTMS points was recorded as $\mathrm{mGMI}_{\mathrm{p}}$, and the mean GMI of language-negative nTMS points as $\mathrm{mGMI}_{n}$ in each patient (Figure 3). Then, intensity extraction was also conducted for the cerebrospinal fluid (CSF) using three randomly selected pixels in the lateral ventricles, and the mean was recorded as $\mathrm{mI}_{\mathrm{csf}}$ to obtain an internal control value (Figure 3). The signal intensity ratio (IR) was calculated as follows [49]:

$$
\begin{aligned}
I R_{p} & =\frac{m G M I_{p}}{m I_{c s f}} \\
I R_{n} & =\frac{m G M I_{n}}{m I_{c s f}}
\end{aligned}
$$




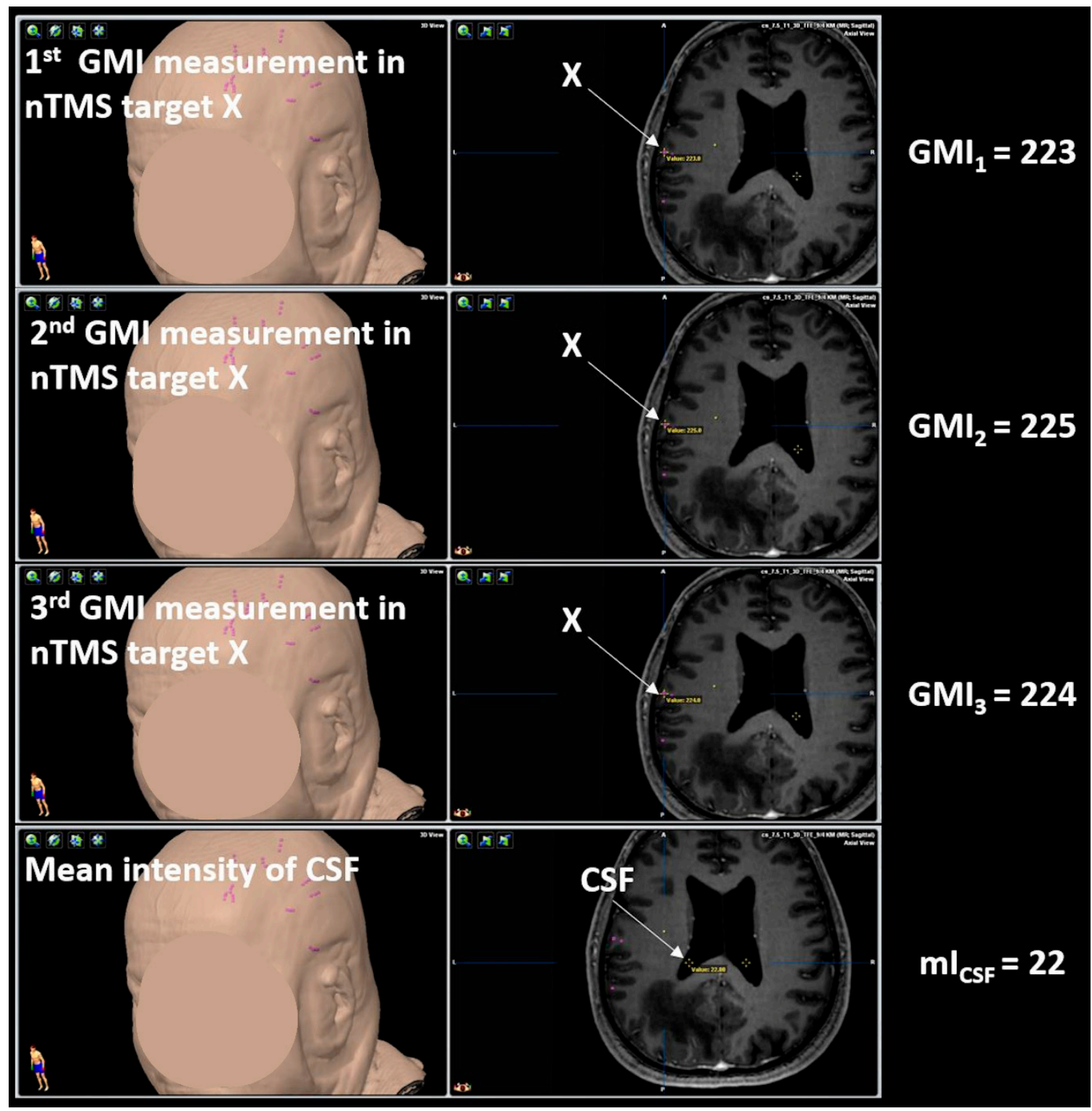

Figure 3. Analysis of gray matter intensity (GMI). The GMI of language-positive or language-negative spots, determined by language mapping using navigated transcranial magnetic stimulation (nTMS), was measured in T1-weighted sequences. Three pixels were randomly selected within a stimulation spot and used to calculate a mean GMI for language-positive nTMS spots $\left(\mathrm{mGMI}_{\mathrm{p}}\right)$ and language-negative nTMS spots $\left(\mathrm{mGMI}_{\mathrm{n}}\right)$. The signal intensity ratio (IR) was then calculated by dividing the $\mathrm{mGMI}_{\mathrm{p}}$ or $\mathrm{mGMI}_{\mathrm{n}}$ by the mean intensity of cerebrospinal fluid $\left(\mathrm{mI}_{\mathrm{csf}}\right)$.

\subsubsection{U-Fibers}

Using nTMS-based DTIFT with the different FAT levels and a minimum FL of 30 and $3 \mathrm{~mm}$, volumes of U-fibers were measured for fiber tractography considering language-positive or language-negative nTMS points as ROIs, respectively (Brainlab Elements, version 3.1.0; Brainlab AG, Munich, Germany; Figure 4). From assessing the difference in volumes between fibers with a minimum FL of $30 \mathrm{~mm}$ and fibers with a minimum FL of $3 \mathrm{~mm}$, the volume of U-fibers was obtained (Figure 4). In this context, U-fibers are considered short association fibers, which connect cortical regions between adjacent gyri and typically have a length in the range of 3 to $30 \mathrm{~mm}$ [48].

A higher number of nTMS points considered during tractography should lead to more fibers, which was corrected for by dividing the fiber volume by $\mathrm{N}_{\mathrm{p}}$ or $\mathrm{N}_{\mathrm{n}}$, resulting in the ratios $\mathrm{R}_{\mathrm{Ufibers} p} p$ and 
$\mathrm{R}_{\text {Ufibers_n }}$ for fibers with a length of 3 to $30 \mathrm{~mm}$. The ratios in $\mathrm{R}_{\text {Ufibers } p p}$ and $\mathrm{R}_{\text {Ufibers_n }}$ were calculated as follows:

$$
\begin{aligned}
& R_{\text {Ufibers_ }_{-}}=\frac{V_{p(3)}-V_{p(30)}}{N_{p}} \\
& R_{\text {Ufibers_n }}=\frac{V_{n(3)}-V_{n(30)}}{N_{n}}
\end{aligned}
$$

$\mathrm{V}_{\mathrm{p}(30)}$ indicates the volume of fibers with a minimum FL of $30 \mathrm{~mm}$, as derived from nTMS-based DTI FT using the ROI of language-positive nTMS points, whereas $V_{p(3)}$ represents the respective volume for a minimum FL of $3 \mathrm{~mm}$. Analogously, $\mathrm{V}_{\mathrm{n}(30)}$ indicates the volume of fibers with a minimum FL of $30 \mathrm{~mm}$, as derived from nTMS-based DTI FT that considers the ROI of language-negative nTMS points, whereas $V_{n(3)}$ represents the respective volume for a minimum FL of $3 \mathrm{~mm}$.

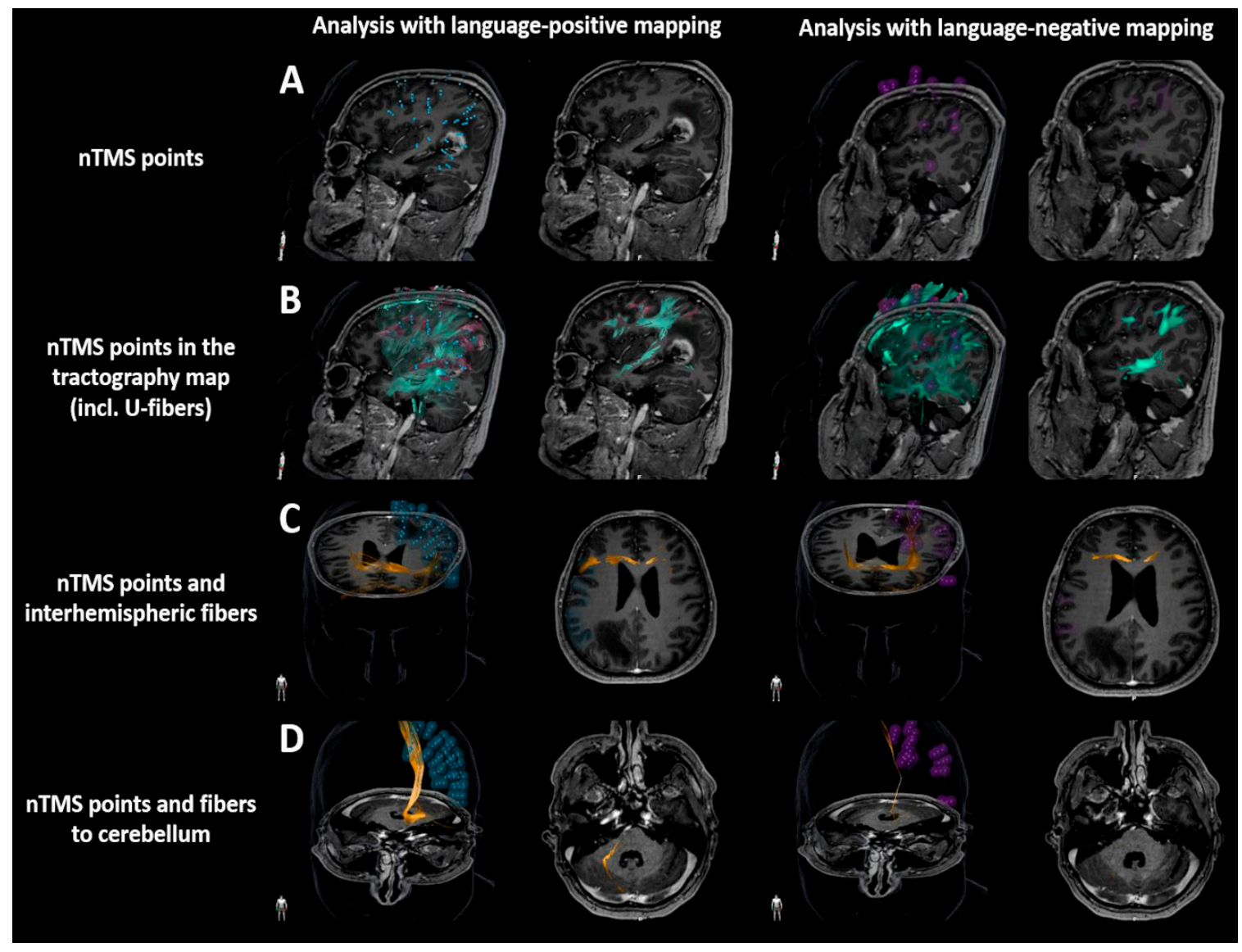

Figure 4. Analysis of subcortical fiber tracts. Tractography maps were generated based on language mapping data derived from navigated transcranial magnetic stimulation (nTMS), using the language-positive nTMS spots and language-negative nTMS spots as separate regions of interest (ROIs). Row (A) visualizes the language-positive nTMS points (blue) and language-negative nTMS points (purple) in an exemplary patient case with a left-hemispheric contrast-enhancing tumor with temporo-parietal location. Row (B) illustrates the complete picture of tractography (green fibers) with special emphasis on U-fibers (purple fibers). Row (C) depicts interhemispheric fiber courses (orange fibers) crossing the midline via the corpus callosum. Row (D) visualizes fibers projecting to the cerebellum (orange fibers).

\subsubsection{Interhemispheric Fibers and Fibers Projecting to the Cerebellum}

The highest percentage of patients presenting Cross-F or Cereb-F according to visual image inspection of tractography maps was achieved for $25 \%$ FAT, which was then taken as the adjustment used for evaluation of Cross-F and Cereb-F volumes, together with a minimum FL of $30 \mathrm{~mm}$ derived 
from language-positive or language-negative nTMS points as ROIs, respectively (Brainlab Elements, version 3.1.0; Brainlab AG, Munich, Germany; Figure 4).

The volumes of the Cross-F and Cereb-F for nTMS-based DTI FT with 25\% FAT were recorded as $\mathrm{V}_{\text {cross } \_p}$ and $\mathrm{V}_{\text {cereb_} \_} p$ when derived from nTMS-based DTI FT using the ROI of language-positive nTMS points, whereas $V_{\text {cross_n }}$ and $V_{\text {cereb_n }}$ represent the Cross-F and Cereb-F volumes for nTMS-based DTI FT conducted with language-negative nTMS points as the ROI. The ratios $\mathrm{R}_{\text {cross_} \_p}$ and $\mathrm{R}_{\text {cereb_} p}$ and $R_{\text {cross_n }}$ and $R_{\text {cereb_n }}$ were calculated as follows:

$$
\begin{aligned}
& R_{\text {cross } \_p}=\frac{V_{\text {cross } \_p}}{N_{p}} \text { and } R_{\text {cereb } \_p}=\frac{V_{\text {cereb } \_p}}{N_{p}} \\
& R_{\text {cross } \_n}=\frac{V_{\text {cross_n }}}{N_{n}} \text { and } R_{\text {cereb } \_n}=\frac{V_{\text {cereb_n }}}{N_{n}}
\end{aligned}
$$

\subsection{Statistical Analyses}

GraphPad Prism (version 6.04; GraphPad Software Inc., La Jolla, CA, USA) was used for statistical data analyses and generation of graphs. Descriptive statistics using relative and absolute frequencies or mean, standard deviation (SD), and ranges were calculated for demographics and characteristics of language mapping and tractography. The Shapiro-Wilk normality test was used to assess the distribution of data, which indicated a non-Gaussian distribution for the majority of data.

To investigate differences between $\mathrm{N}_{\mathrm{p}}$ and $\mathrm{N}_{\mathrm{n}}, \mathrm{IR}_{\mathrm{p}}$ and $\mathrm{IR}_{\mathrm{n}}, \mathrm{R}_{\text {Ufibers_p } p}$ and $\mathrm{R}_{\mathrm{Ufiber} \_n}$ (separately for nTMS-based DTI FT with $100 \%$ FAT, 75\% FAT, 50\% FAT, and 25\% FAT), $R_{\text {cross_p }}$ and $R_{\text {cross_n }}$, and $R_{\text {cereb_p }}$ and $R_{\text {cereb_n }}$, Wilcoxon matched-pairs signed rank tests were performed. Furthermore, correlation analyses computing Spearman's rho were performed between $\mathrm{N}_{\mathrm{p}}, \mathrm{IR}_{\mathrm{p}}, \mathrm{R}_{\mathrm{Ufibers} \_}, \mathrm{R}_{\text {cross_p}}$, and $\mathrm{R}_{\text {cereb_} p}$ as well as, analogously, between $N_{n}, I_{n}, R_{U f i b e r s \_n}, R_{\text {cross_n}}$, and $R_{\text {cereb_n }}$ and the status of preoperative, postoperative, and follow-up aphasia, considering the four grades as derived from language function assessments at different time points. The correlation analyses were adjusted for multiple testing using the Benjamini-Hochberg procedure with a false discovery rate of $25 \%$.

\section{Results}

\subsection{Cohort Characteristics}

Twenty patients (mean age: $63.2 \pm 12.9$ years, age range: $20.3-80.8$ years, 4 females and 16 males, 16 right-handers according to EHI scores) were included, all diagnosed with a left-hemispheric GBM according to histopathological evaluation (Table 1$)$. Ten patients (50\%) showed preoperative aphasia, whereas eight patients (40\%) showed aphasia during follow-up examinations three months after tumor resection. GTR according to postoperative MRI was achieved in 10 patients (50\%).

\subsection{Comparison between Language-Positive and Language-Negative Mapping and Tractography}

Language mapping of the tumor-affected LH and nTMS-based DTI FT was possible in all enrolled patients. None of the patients showed adverse events in the course of stimulation.

There were statistically significant differences in almost all measures between mapping or tractography using language-positive or language-negative nTMS points as ROIs, respectively (Table 2). In detail, patients showed a higher mean number of language-negative nTMS points $(p=0.0026)$, whereas the IR of these points was elevated on average in comparison to language-positive nTMS points ( $p=0.0121$; Table 2). The ratios for U-fiber volumes as well as for long fibers projecting to the contralateral hemisphere and fibers projecting to the cerebellum were higher for tractography using language-positive nTMS points ( $p \leq 0.0494$; Table 2). 
Table 1. Cohort characteristics.

\begin{tabular}{|c|c|c|c|c|c|c|c|c|c|c|}
\hline \multirow{2}{*}{ No. } & \multirow{2}{*}{ Sex } & \multirow{2}{*}{ Age } & \multirow{2}{*}{$\begin{array}{l}\text { No. of } \\
\text { Language-Positive } \\
\text { CPS Sites }\end{array}$} & \multirow{2}{*}{$\begin{array}{c}\text { No. of } \\
\text { Language-Negative } \\
\text { CPS Sites }\end{array}$} & \multirow{2}{*}{$\begin{array}{l}\text { 100\% FAT for DTI FT with } \\
\text { Language-Positive nTMS Points }\end{array}$} & \multirow{2}{*}{$\begin{array}{c}100 \% \text { FAT for DTI FT with } \\
\text { Language-Negative nTMS Points }\end{array}$} & \multirow{2}{*}{$\begin{array}{l}\text { Awake } \\
\text { Surgery }\end{array}$} & \multicolumn{3}{|c|}{ Aphasia Grading } \\
\hline & & & & & & & & Preop & Postop & Follow-up \\
\hline 1 & male & 52.0 & 12 & 34 & 0.29 & 0.32 & No & 0 & 0 & 0 \\
\hline 2 & male & 76.7 & 18 & 26 & 0.32 & 0.34 & No & 2 & 2 & 1 \\
\hline 3 & male & 68.5 & 15 & 30 & 0.39 & 0.39 & Yes & 0 & 0 & 0 \\
\hline 4 & male & 54.2 & 15 & 31 & 0.42 & 0.48 & Yes & 2 & 0 & 0 \\
\hline 5 & male & 52.2 & 8 & 38 & 0.42 & 0.36 & No & 1 & 1 & 1 \\
\hline 6 & male & 57.0 & 10 & 30 & 0.49 & 0.58 & No & 0 & 0 & 0 \\
\hline 7 & female & 70.1 & 12 & 34 & 0.29 & 0.32 & No & 0 & 0 & 0 \\
\hline 8 & male & 64.3 & 31 & 15 & 0.39 & 0.39 & No & 3 & 3 & 3 \\
\hline 9 & male & 72.6 & 5 & 41 & 0.33 & 0.38 & No & 0 & 0 & 0 \\
\hline 10 & male & 57.3 & 8 & 35 & 0.29 & 0.38 & Yes & 1 & 1 & 1 \\
\hline 11 & male & 71.5 & 35 & 11 & 0.33 & 0.37 & Yes & 2 & 2 & 2 \\
\hline 12 & male & 55.6 & 28 & 18 & 0.39 & 0.35 & No & 0 & 0 & 0 \\
\hline 13 & female & 72.2 & 9 & 35 & 0.34 & 0.35 & No & 1 & 0 & 0 \\
\hline 14 & male & 74.4 & 24 & 19 & 0.37 & 0.36 & No & 0 & 2 & 0 \\
\hline 15 & male & 70.6 & 16 & 29 & 0.37 & 0.36 & No & 1 & 2 & 1 \\
\hline 16 & male & 62.0 & 12 & 33 & 0.33 & 0.43 & No & 0 & 0 & 0 \\
\hline 17 & female & 60.5 & 19 & 25 & 0.37 & 0.43 & Yes & 2 & 2 & 2 \\
\hline 18 & male & 20.3 & 2 & 38 & 0.38 & 0.44 & No & 0 & 0 & 0 \\
\hline 19 & female & 80.8 & 5 & 38 & 0.30 & 0.28 & No & 0 & 0 & 0 \\
\hline 20 & male & 70.3 & 18 & 28 & 0.41 & 0.37 & No & 2 & 3 & 3 \\
\hline
\end{tabular}

This table shows cohort details, including sex distribution and age (in years), information on the number of language-positive and language-negative sites according to the cortical parcellation system (CPS), the fractional anisotropy threshold (FAT) for diffusion tensor imaging fiber tracking (DTI FT) based on navigated transcranial stimulation (nTMS), and aphasia grades (at three different time points: preop = preoperative status, postop = postoperative status, follow-up = status during follow-up examinations three months after surgery). Language mapping aimed to cover 46 target points in total that were placed in relation to the CPS on the left hemisphere (LH), but a reduced number of targets was stimulated in patients with large tumor masses that precluded placement of all 46 target points. Thus, the numbers of language-positive and language-negative CPS sites do not necessarily add up to 46 in all enrolled patients. 
Table 2. Comparison between language-positive and language-negative mapping and tractography.

\begin{tabular}{|c|c|c|c|c|c|c|}
\hline & \multirow{2}{*}{ Item } & \multicolumn{2}{|c|}{$\begin{array}{l}\text { Language-Positive } \\
\text { nTMS Points }\end{array}$} & \multicolumn{2}{|c|}{$\begin{array}{l}\text { Language-Negative } \\
\text { nTMS Points }\end{array}$} & \multirow[t]{2}{*}{$p$} \\
\hline & & Mean & SD & Mean & SD & \\
\hline \multirow{6}{*}{$\mathrm{R}_{\text {Ufibers }}$} & $\mathrm{N}$ & 15.1 & 8.9 & 29.4 & 8.3 & 0.0026 \\
\hline & IR & 5.7 & 1.7 & 7.1 & 1.6 & 0.0121 \\
\hline & $100 \%$ FAT & 0.3 & 0.2 & 0.1 & 0.1 & 0.0012 \\
\hline & $75 \%$ FAT & 0.5 & 0.2 & 0.3 & 0.3 & 0.0020 \\
\hline & $50 \%$ FAT & 0.6 & 0.3 & 0.4 & 0.2 & 0.0056 \\
\hline & $25 \%$ FAT & 0.7 & 0.5 & 0.4 & 0.3 & 0.1231 \\
\hline \multirow{2}{*}{\multicolumn{2}{|c|}{$\begin{array}{l}\mathrm{R}_{\text {cross }} \\
\mathrm{R}_{\text {cereb }}\end{array}$}} & 0.9 & 0.8 & 0.5 & 0.6 & 0.0494 \\
\hline & & 0.6 & 0.6 & 0.3 & 0.5 & 0.0094 \\
\hline
\end{tabular}

This table shows the mean and standard deviation (SD) for the number (N) of language-positive and language-negative points as mapped by navigated transcranial magnetic stimulation (nTMS), intensity ratio (IR), ratio of volumes for U-fibers ( $R_{\text {Ufibers, }}$, as derived from tractography using $100 \%, 75 \%, 50 \%$, and $25 \%$ of the individual fractional anisotropy threshold (FAT)), and ratio of volumes for interhemispheric fibers ( $\mathrm{R}_{\text {cross }}, \mathrm{using}$ tractography with $25 \%$ FAT) as well as fibers projecting to the cerebellum ( $R_{\text {cereb }}$, using tractography with $25 \%$ FAT). Wilcoxon matched-pairs signed rank tests were conducted to assess differences in these characteristics between language-positive and language-negative mappings (level of statistical significance: $p<0.05$ ). Statistically significant values are displayed in bold.

\subsection{Associations with Aphasia Grading}

For language-positive nTMS points, statistically significant positive correlations were revealed between their absolute frequency and aphasia for the preoperative $(R=0.4919, p=0.0276)$, postoperative $(\mathrm{R}=0.6183, p=0.0037)$, and follow-up status $(\mathrm{R}=0.4854, p=0.0300$; Table 3$)$. The higher the number of language-positive nTMS points of the tumor-affected LH, the higher the aphasia grade. Furthermore, statistically significant negative correlations were observed between the ratio of U-fiber volumes (considering tractography with 100\% FAT) and aphasia for the postoperative $(\mathrm{R}=-0.6102, p=0.0043$ ) as well as follow-up status ( $\mathrm{R}=-0.4899, p=0.0283$; Table 3$)$. Thus, the lower this ratio was, the higher the aphasia grade.

Regarding language-negative nTMS points, statistically significant negative correlations were revealed between their absolute frequency and aphasia for postoperative $(\mathrm{R}=-0.6097, p=0.0043)$ and follow-up examinations $(\mathrm{R}=-0.4741, p=0.0347$; Table 3). Hence, the higher the number of language-negative nTMS points of the tumor-affected LH, the lower the aphasia grade.

Table 3. Associations with aphasia grading.

\begin{tabular}{|c|c|c|c|c|c|c|}
\hline \multirow{2}{*}{ ROI } & \multirow{2}{*}{\multicolumn{2}{|c|}{ Item }} & \multirow{2}{*}{ Parameter } & \multicolumn{3}{|c|}{ Aphasia Grading } \\
\hline & & & & Preoperative & Postoperative & Follow-Up \\
\hline \multirow{16}{*}{$\begin{array}{l}\text { Language-Positive } \\
\text { nTMS Points }\end{array}$} & \multirow{2}{*}{\multicolumn{2}{|c|}{$\mathrm{N}$}} & rho & 0.4919 & 0.6183 & 0.4854 \\
\hline & & & $p$ & 0.0276 & 0.0037 & 0.0300 \\
\hline & \multirow{2}{*}{\multicolumn{2}{|c|}{ IR }} & rho & 0.0138 & -0.0806 & 0.0888 \\
\hline & & & $p$ & 0.9538 & 0.7354 & 0.7098 \\
\hline & \multirow{2}{*}{\multicolumn{2}{|c|}{$100 \%$ FAT }} & rho & -0.3777 & -0.6102 & -0.4899 \\
\hline & & & $p$ & 0.1007 & 0.0043 & 0.0283 \\
\hline & \multirow{2}{*}{\multicolumn{2}{|c|}{$75 \%$ FAT }} & rho & -0.2645 & -0.4323 & -0.4080 \\
\hline & & & $p$ & 0.2597 & 0.0570 & 0.0741 \\
\hline & NUfibers & \multirow{2}{*}{$50 \%$ FAT } & rho & -0.0016 & 0.0590 & -0.0854 \\
\hline & & & $p$ & 0.9946 & 0.8048 & 0.7205 \\
\hline & & \multirow{2}{*}{$25 \%$ FAT } & rho & -0.1823 & 0.0341 & -0.0632 \\
\hline & & & $p$ & 0.4417 & 0.8866 & 0.7914 \\
\hline & \multirow{2}{*}{\multicolumn{2}{|c|}{$\mathrm{R}_{\text {cross }}$}} & rho & -0.4590 & -0.1629 & -0.2288 \\
\hline & & & $p$ & 0.0418 & 0.4925 & 0.3320 \\
\hline & \multirow{2}{*}{\multicolumn{2}{|c|}{$\mathrm{R}_{\text {cereb }}$}} & rho & -0.1717 & -0.1347 & -0.1110 \\
\hline & & & $p$ & 0.4691 & 0.5713 & 0.6414 \\
\hline
\end{tabular}


Table 3. Cont.

\begin{tabular}{|c|c|c|c|c|c|c|}
\hline \multirow{2}{*}{ ROI } & \multirow{2}{*}{\multicolumn{2}{|c|}{ Item }} & \multirow{2}{*}{ Parameter } & \multicolumn{3}{|c|}{ Aphasia Grading } \\
\hline & & & & Preoperative & Postoperative & Follow-Up \\
\hline \multirow{16}{*}{$\begin{array}{c}\text { Language-Negative } \\
\text { nTMS Points }\end{array}$} & \multirow{3}{*}{\multicolumn{2}{|c|}{$\mathrm{N}$}} & rho & -0.4521 & -0.6097 & -0.4741 \\
\hline & & & $p$ & 0.0454 & 0.0043 & 0.0347 \\
\hline & \multirow{3}{*}{\multicolumn{2}{|c|}{ IR }} & rho & -0.1660 & -0.1987 & -0.2475 \\
\hline & & & $p$ & 0.4842 & 0.4011 & 0.2927 \\
\hline & & & rho & 0.0733 & -0.1297 & -0.0956 \\
\hline & \multirow{7}{*}{$\mathrm{R}_{\text {Ufibers }}$} & $100 \%$ FAT & $p$ & 0.7589 & 0.5858 & 0.6885 \\
\hline & & \multirow{2}{*}{$75 \%$ FAT } & rho & -0.2784 & -0.2752 & -0.3141 \\
\hline & & & $p$ & 0.2347 & 0.2403 & 0.1774 \\
\hline & & \multirow{2}{*}{$50 \%$ FAT } & rho & -0.1457 & 0.1621 & 0.0768 \\
\hline & & & $p$ & 0.5400 & 0.4947 & 0.7475 \\
\hline & & \multirow{2}{*}{$25 \%$ FAT } & rho & 0.1750 & 0.3267 & 0.2885 \\
\hline & & & $p$ & 0.4606 & 0.1598 & 0.2174 \\
\hline & \multirow{2}{*}{\multicolumn{2}{|c|}{$\mathrm{R}_{\text {cross }}$}} & rho & 0.0073 & -0.0399 & 0.0598 \\
\hline & & & $p$ & 0.9755 & 0.8674 & 0.8024 \\
\hline & \multirow{2}{*}{\multicolumn{2}{|c|}{$\mathrm{R}_{\text {cereb }}$}} & rho & 0.0887 & 0.2959 & 0.1912 \\
\hline & & & $p$ & 0.7099 & 0.2052 & 0.4194 \\
\hline
\end{tabular}

This table shows the correlation results between the number of language-positive and language-negative points as mapped by navigated transcranial magnetic stimulation (nTMS), intensity ratio (IR), ratio of volumes for U-fibers ( $\mathrm{R}_{\text {Ufibers, }}$ as derived from tractography using 100\%, 75\%,50\%, and 25\% of the individual fractional anisotropy threshold (FAT)), ratio of volumes for interhemispheric fibers ( $R_{\text {cross, }}$ using tractography with $25 \%$ FAT) as well as fibers projecting to the cerebellum $\left(R_{\text {cereb, }}\right.$ using tractography with $25 \%$ FAT) and the aphasia grades for the preoperative, postoperative, and follow-up status. Correlation coefficients are represented by Spearman's rho, and related $p$-values are given (level of statistical significance: $p<0.05)$. Statistically significant values that survived adjustments for multiple testing (Benjamini-Hochberg procedure with a false discovery rate of $25 \%$ ) are depicted in bold.

\section{Discussion}

This study investigated the difference between language-positive and language-negative mappings as derived from presurgical nTMS and nTMS-based DTI FT in patients harboring supratentorial GBMs. There are three main results that can be taken from our analyses. First, regarding the cortical level, a significantly lower GMI was revealed for language-positive nTMS points compared to language-negative counterparts. Second, on the subcortical level, language-positive areas were characterized by an increased connectivity profile, i.e., such areas showed a significantly higher ratio in volumes for U-fibers, interhemispheric fibers, and fibers projecting to the cerebellum. Third, the number of language-positive nTMS points as well as the ratio in volumes for U-fibers were significantly associated with aphasia grading as derived from assessments at different time points.

\subsection{Gray Matter Intensity}

While there is evidence for alterations in GM distribution and volume related to language function, the role of the GMI to characterize language-involved areas has not been investigated to the authors' knowledge. Specifically, previous research has detected that language lateralization is predicted by the degree of GM lateralization [30]. Further, subjects diagnosed with dyslexia showed GM deficits, but the GM volume, however, can be subject to changes following training interventions in dyslexic children [31,50]. On the contrary, region-specific increases in GM volume have also been revealed for developmental language disorders, which might be interpreted as a result of compensatory mechanisms [31,32]. In the present study, lower GMI in T1-weighted sequences was revealed for language-positive nTMS points when compared to language-negative spots. This may probably reflect a sign of higher GM density, potentially suggesting increased functional involvement. Yet, this remains speculative until further studies using a similar setup can confirm our findings. For the present study, whether potentially increased functional involvement is due to higher intrinsic contribution of such areas to language function or related to compensatory mechanisms for language function at risk in our sample remains beyond the scope of investigation. However, the findings for GMI may 
provide a direct link between a functionally language-related area as mapped by nTMS and structural cortical characteristics.

\subsection{White Matter Tractography}

The ratios for fiber volumes of U-fibers, transcallosal fibers, and fibers coursing to the cerebellum were higher for language-positive areas compared to language-negative counterparts. Thus, language-related spots seem to be characterized by an enhanced connectivity profile, which exists focally for short fibers connecting cortical regions between adjacent gyri (increased $\mathrm{R}_{\text {Ufibers }}$ ) as well as more remotely for long connecting fibers (increased $R_{\text {cross }}$ and $R_{\text {cereb}}$ ). This may reflect either higher a-priori involvement in the cortico-subcortical language network, compensatory mechanisms in light of language function at risk in brain tumor patients, or a mixed picture of both. While the specificity of nTMS language mapping in comparison to intraoperative DES has shown to be comparatively low with $23.8 \%$, the technique's negative predictive value was $83.9 \%$, implicating that language-negative nTMS points are also mostly negative in intraoperative DES mapping [13]. The significantly lower connectivity profile of language-negative nTMS spots as revealed by the present study may resemble the "truly" absent or less involved character of these spots. Furthermore, a previous study on nTMS-based DTI FT revealed that there is a higher likelihood of subcortical connections with language-positive nTMS spots as compared to language-negative ones, with true-positive connections (connections for positive spots) being visualized up to four-fold more frequently than false-positive connections (connections for negative spots) [21]. The clear difference in this likelihood could reflect high reliability of nTMS-based DTI FT for the purpose of tracking parts of the human language network [21]. Likewise, the enhanced connectivity profile to adjacent gyri as well as the contralateral hemisphere and cerebellum may serve as a surrogate of good reliability.

\subsection{Associations with Aphasia}

The number of language-positive nTMS points was significantly associated with aphasia grading. The higher the aphasia grading is (i.e., the more severe language impairment is), the higher the number of language-positive nTMS spots. A higher frequency of language-positive nTMS spots among more impaired patients according to pre- and postoperative as well as follow-up examinations makes sense as a decline in language function should be associated with higher stimulation-induced errors, although this may bias the results of nTMS language mapping. A previous study is in good accordance with this finding and showed that aphasia as measured by the Berlin Aphasia Score correlated significantly with the incidence of errors during nTMS language mapping; yet, correlations were only evaluated for the preoperative status of language function [51]. Moreover, higher aphasia grading for the postoperative and follow-up status was associated with lower ratios in volume for U-fibers. Hence, this finding may underline the important role of short association fibers for keeping of language function as their impairment, e.g., in the direct perioperative course of tumor resection or related to even subtle perioperative ischemia, could result in language worsening.

\subsection{Limitations and Perspectives}

When interpreting the results of this study, the following limitations have to be acknowledged. First, the retrospective character and comparatively small sample size restrict the generalizability of the findings. Upcoming studies may include more patients and follow a prospective study design. Second, the finding of lower GMI for language-positive nTMS points when compared to language-negative nTMS points and related interpretation as a potential hint for higher GM density needs further validation. A potential explanation could also be linked to the increased connectivity profile of language-positive nTMS points. Future studies using imaging with higher resolution may provide evidence for our preliminary interpretations. Third, the technique of DTI has its inherent methodological shortcomings, which could lead to aberrant fiber reconstruction and visualization, particularly for crossing or kissing fibers and in the presence of edema, which is commonly observed 
in relation to brain tumors [52,53]. Other sequences and tracking algorithms are developed to become applicable in the clinical setting, but good alternatives to conventional DTI and its tractography-based analyses are still not available for clinical routine use [54,55]. Fourth, the tractography maps generated by nTMS-based DTI FT for depiction of the language network need further validation, ideally by intraoperative DES as the gold-standard method. In this context, previous studies have evaluated the agreement between preoperative nTMS language mapping and intraoperative DES [13,16,24]; however, on the subcortical level, such correlation analyses in representative samples are largely missing to date. First evidence of associations between preoperative nTMS-based tractography and intraoperative DES results or surgery-related aphasia has been obtained [20,37]. Upcoming studies may use more sophisticated approaches for diffusion-weighted MRI and fiber tractography, ideally combining it with functional data such as nTMS maps after further confirmatory studies. Furthermore, repeated investigations of the language network by serial nTMS language mappings and tractography after surgery may be of interest to track potential plastic effects as well as associations with aphasia grades on a longitudinal scale.

\title{
5. Conclusions
}

Language-positive and language-negative areas as determined by nTMS language mapping show differences in cortical and subcortical characteristics among patients diagnosed with GBMs. Specifically, language-positive areas demonstrate lower GMI and an enhanced connectivity profile with higher volume ratios for U-fibers, interhemispheric fibers, and fibers projecting to the cerebellum. While future studies may confirm these results in larger cohorts in the context of a prospective study design, these findings facilitate confidence in the technique of nTMS language mapping and nTMS-based tractography to detect language-involved structures of the human cortico-subcortical language network.

Author Contributions: Conceptualization, H.Z., S.S. and N.S.; methodology, H.Z., S.S., S.M.K. and N.S.; software, H.Z.; validation, S.M.K. and N.S.; formal analysis, H.Z., S.S., A.S. and N.S.; investigation, H.Z., S.S. and A.S.; resources, C.Z., B.M. and S.M.K.; data curation, H.Z. and N.S.; writing-original draft preparation, H.Z. and N.S.; writing-review and editing, S.M.K.; visualization, H.Z.; supervision, C.Z., B.M., S.M.K. and N.S.; project administration, S.M.K. and N.S.; funding acquisition, C.Z., B.M. and S.M.K. All authors have read and agreed to the published version of the manuscript.

Funding: This research received no external funding.

Conflicts of Interest: N.S. received honoraria from Nexstim Plc (Helsinki, Finland). S.M.K. is a consultant for Nexstim Plc (Helsinki, Finland) and Spineart Deutschland GmbH (Frankfurt, Germany) and received honoraria from Medtronic (Meerbusch, Germany) and Carl Zeiss Meditec (Oberkochen, Germany). S.M.K. and B.M. received research grants and are consultants for Brainlab AG (Munich, Germany). B.M. received honoraria, consulting fees, and research grants from Medtronic (Meerbusch, Germany), Icotec ag (Altstätten, Switzerland), and Relievant Medsystems Inc. (Sunnyvale, CA, USA); honoraria and research grants from Ulrich Medical (Ulm, Germany); honoraria and consulting fees from Spineart Deutschland GmbH (Frankfurt, Germany) and DePuy Synthes (West Chester, PA, USA); and royalties from Spineart Deutschland GmbH (Frankfurt, Germany). The other authors declare no conflicts of interest.

\author{
Abbreviations \\ CPS Cortical parcellation system \\ CSF Cerebrospinal fluid \\ DES Direct electrical stimulation \\ DICOM Digital Imaging and Communications in Medicine \\ DTI Diffusion tensor imaging \\ DTI FT Diffusion tensor imaging fiber tracking \\ EHI Edinburgh Handedness Inventory \\ FA Fractional anisotropy \\ FAT Fractional anisotropy threshold \\ FL Fiber length
}




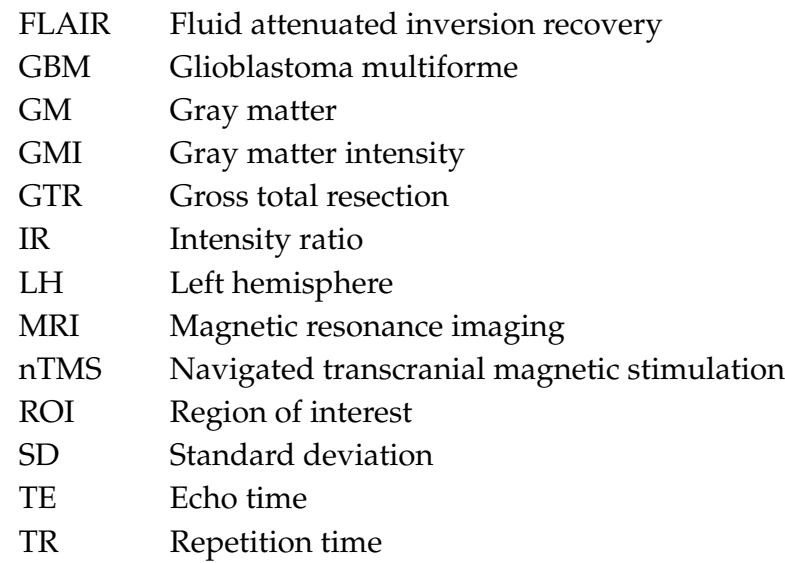

\section{References}

1. Duffau, H.; Mandonnet, E. The "onco-functional balance" in surgery for diffuse low-grade glioma: Integrating the extent of resection with quality of life. Acta Neurochir. 2013, 155, 951-957. [CrossRef]

2. Sanai, N.; Berger, M.S. Glioma extent of resection and its impact on patient outcome. Neurosurgery 2008, 62, 753-766. [CrossRef]

3. Eyupoglu, I.Y.; Buchfelder, M.; Savaskan, N.E. Surgical resection of malignant gliomas-role in optimizing patient outcome. Nat. Rev. Neurol. 2013, 9, 141-151. [CrossRef] [PubMed]

4. Hervey-Jumper, S.L.; Berger, M.S. Maximizing safe resection of low- and high-grade glioma. J. Neuro-Oncol. 2016, 130, 269-282. [CrossRef] [PubMed]

5. Schucht, P.; Beck, J.; Seidel, K.; Raabe, A. Extending resection and preserving function: Modern concepts of glioma surgery. Swiss Med. Wkly. 2015, 145, w14082. [CrossRef] [PubMed]

6. Ottenhausen, M.; Krieg, S.M.; Meyer, B.; Ringel, F. Functional preoperative and intraoperative mapping and monitoring: Increasing safety and efficacy in glioma surgery. Neurosurg. Focus 2015, 38, E3. [CrossRef] [PubMed]

7. Sanai, N.; Berger, M.S. Mapping the horizon: Techniques to optimize tumor resection before and during surgery. Clin. Neurosurg. 2008, 55, 14-19. [PubMed]

8. Duffau, H.; Lopes, M.; Arthuis, F.; Bitar, A.; Sichez, J.P.; Van Effenterre, R.; Capelle, L. Contribution of intraoperative electrical stimulations in surgery of low grade gliomas: A comparative study between two series without (1985-96) and with (1996-2003) functional mapping in the same institution. J. Neurol. Neurosurg. Psychiatry 2005, 76, 845-851. [CrossRef] [PubMed]

9. De Witt Hamer, P.C.; Robles, S.G.; Zwinderman, A.H.; Duffau, H.; Berger, M.S. Impact of intraoperative stimulation brain mapping on glioma surgery outcome: A meta-analysis. J. Clin. Oncol. Off. J. Am. Soc. Clin. Oncol. 2012, 30, 2559-2565. [CrossRef]

10. Mandonnet, E.; Winkler, P.A.; Duffau, H. Direct electrical stimulation as an input gate into brain functional networks: Principles, advantages and limitations. Acta Neurochir. 2010, 152, 185-193. [CrossRef]

11. Ruohonen, J.; Karhu, J. Navigated transcranial magnetic stimulation. Neurophysiol. Clin. Clin. Neurophysiol. 2010, 40, 7-17. [CrossRef]

12. Picht, T. Current and potential utility of transcranial magnetic stimulation in the diagnostics before brain tumor surgery. CNS Oncol. 2014, 3, 299-310. [CrossRef]

13. Picht, T.; Krieg, S.M.; Sollmann, N.; Rosler, J.; Niraula, B.; Neuvonen, T.; Savolainen, P.; Lioumis, P.; Makela, J.P.; Deletis, V.; et al. A comparison of language mapping by preoperative navigated transcranial magnetic stimulation and direct cortical stimulation during awake surgery. Neurosurgery 2013, 72, 808-819. [CrossRef]

14. Sollmann, N.; Kelm, A.; Ille, S.; Schroder, A.; Zimmer, C.; Ringel, F.; Meyer, B.; Krieg, S.M. Setup presentation and clinical outcome analysis of treating highly language-eloquent gliomas via preoperative navigated transcranial magnetic stimulation and tractography. Neurosurg. Focus 2018, 44, E2. [CrossRef] [PubMed]

15. Sollmann, N.; Meyer, B.; Krieg, S.M. Implementing Functional Preoperative Mapping in the Clinical Routine of a Neurosurgical Department: Technical Note. World Neurosurg. 2017, 103, 94-105. [CrossRef] [PubMed] 
16. Tarapore, P.E.; Findlay, A.M.; Honma, S.M.; Mizuiri, D.; Houde, J.F.; Berger, M.S.; Nagarajan, S.S. Language mapping with navigated repetitive TMS: Proof of technique and validation. NeuroImage 2013, 82, 260-272. [CrossRef] [PubMed]

17. Sollmann, N.; Negwer, C.; Ille, S.; Maurer, S.; Hauck, T.; Kirschke, J.S.; Ringel, F.; Meyer, B.; Krieg, S.M. Feasibility of nTMS-based DTI fiber tracking of language pathways in neurosurgical patients using a fractional anisotropy threshold. J. Neurosci. Methods 2016, 267, 45-54. [CrossRef] [PubMed]

18. Negwer, C.; Ille, S.; Hauck, T.; Sollmann, N.; Maurer, S.; Kirschke, J.S.; Ringel, F.; Meyer, B.; Krieg, S.M. Visualization of subcortical language pathways by diffusion tensor imaging fiber tracking based on rTMS language mapping. Brain Imaging Behav. 2017, 11, 899-914. [CrossRef] [PubMed]

19. Sollmann, N.; Zhang, H.; Schramm, S.; Ille, S.; Negwer, C.; Kreiser, K.; Meyer, B.; Krieg, S.M. Function-specific Tractography of Language Pathways Based on nTMS Mapping in Patients with Supratentorial Lesions. Clin Neuroradiol. 2018. [CrossRef] [PubMed]

20. Sollmann, N.; Giglhuber, K.; Tussis, L.; Meyer, B.; Ringel, F.; Krieg, S.M. nTMS-based DTI fiber tracking for language pathways correlates with language function and aphasia-A case report. Clin. Neurol. Neurosurg. 2015, 136, 25-28. [CrossRef] [PubMed]

21. Raffa, G.; Bahrend, I.; Schneider, H.; Faust, K.; Germano, A.; Vajkoczy, P.; Picht, T. A Novel Technique for Region and Linguistic Specific nTMS-based DTI Fiber Tracking of Language Pathways in Brain Tumor Patients. Front. Neurosci. 2016, 10, 552. [CrossRef] [PubMed]

22. Raffa, G.; Conti, A.; Scibilia, A.; Sindorio, C.; Quattropani, M.C.; Visocchi, M.; Germano, A.; Tomasello, F. Functional Reconstruction of Motor and Language Pathways Based on Navigated Transcranial Magnetic Stimulation and DTI Fiber Tracking for the Preoperative Planning of Low Grade Glioma Surgery: A New Tool for Preservation and Restoration of Eloquent Networks. Acta Neurochir. Suppl. 2017, 124, 251-261. [CrossRef] [PubMed]

23. Sollmann, N.; Zhang, H.; Fratini, A.; Wildschuetz, N.; Ille, S.; Schroder, A.; Zimmer, C.; Meyer, B.; Krieg, S.M. Risk Assessment by Presurgical Tractography Using Navigated TMS Maps in Patients with Highly Motor- or Language-Eloquent Brain Tumors. Cancers 2020, 12, 1264. [CrossRef] [PubMed]

24. Sollmann, N.; Kubitscheck, A.; Maurer, S.; Ille, S.; Hauck, T.; Kirschke, J.S.; Ringel, F.; Meyer, B.; Krieg, S.M. Preoperative language mapping by repetitive navigated transcranial magnetic stimulation and diffusion tensor imaging fiber tracking and their comparison to intraoperative stimulation. Neuroradiology 2016, 58, 807-818. [CrossRef]

25. Sollmann, N.; Negwer, C.; Tussis, L.; Hauck, T.; Ille, S.; Maurer, S.; Giglhuber, K.; Bauer, J.S.; Ringel, F.; Meyer, B.; et al. Interhemispheric connectivity revealed by diffusion tensor imaging fiber tracking derived from navigated transcranial magnetic stimulation maps as a sign of language function at risk in patients with brain tumors. J. Neurosurg. 2017, 126, 222-233. [CrossRef] [PubMed]

26. Chang, E.F.; Raygor, K.P.; Berger, M.S. Contemporary model of language organization: An overview for neurosurgeons. J. Neurosurg. 2015, 122, 250-261. [CrossRef] [PubMed]

27. Fedorenko, E.; Thompson-Schill, S.L. Reworking the language network. Trends Cogn. Sci. 2014, 18, 120-126. [CrossRef]

28. Dick, A.S.; Bernal, B.; Tremblay, P. The language connectome: New pathways, new concepts. Neuroscientist 2014, 20, 453-467. [CrossRef]

29. Yagmurlu, K.; Middlebrooks, E.H.; Tanriover, N.; Rhoton, A.L., Jr. Fiber tracts of the dorsal language stream in the human brain. J. Neurosurg. 2016, 124, 1396-1405. [CrossRef]

30. Josse, G.; Kherif, F.; Flandin, G.; Seghier, M.L.; Price, C.J. Predicting language lateralization from gray matter. J. Neurosci. Off. J. Soc. Neurosci. 2009, 29, 13516-13523. [CrossRef]

31. Vinckenbosch, E.; Robichon, F.; Eliez, S. Gray matter alteration in dyslexia: Converging evidence from volumetric and voxel-by-voxel MRI analyses. Neuropsychologia 2005, 43, 324-331. [CrossRef] [PubMed]

32. Pigdon, L.; Willmott, C.; Reilly, S.; Conti-Ramsden, G.; Gaser, C.; Connelly, A.; Morgan, A.T. Grey matter volume in developmental speech and language disorder. Brain Struct. Funct. 2019, 224, 3387-3398. [CrossRef]

33. Steinmann, S.; Mulert, C. Functional relevance of interhemispheric fiber tracts in speech processing. J. Neurolinguistics 2012, 25, 1-12. [CrossRef]

34. Fabbro, F. Introduction to language and cerebellum. J. Neurolinguistics 2000, 13, 83-94. [CrossRef]

35. Huber, W.; Poeck, K.; Willmes, K. The Aachen Aphasia Test. Adv. Neurol. 1984, 42, 291-303. [PubMed] 
36. Sollmann, N.; Ille, S.; Hauck, T.; Maurer, S.; Negwer, C.; Zimmer, C.; Ringel, F.; Meyer, B.; Krieg, S.M. The impact of preoperative language mapping by repetitive navigated transcranial magnetic stimulation on the clinical course of brain tumor patients. BMC Cancer 2015, 15, 261. [CrossRef] [PubMed]

37. Negwer, C.; Beurskens, E.; Sollmann, N.; Maurer, S.; Ille, S.; Giglhuber, K.; Kirschke, J.S.; Ringel, F.; Meyer, B.; Krieg, S.M. Loss of Subcortical Language Pathways Correlates with Surgery-Related Aphasia in Patients with Brain Tumor: An Investigation via Repetitive Navigated Transcranial Magnetic Stimulation-Based Diffusion Tensor Imaging Fiber Tracking. World Neurosurg. 2018, 111, e806-e818. [CrossRef]

38. Oldfield, R.C. The assessment and analysis of handedness: The Edinburgh inventory. Neuropsychologia 1971, 9, 97-113. [CrossRef]

39. Ruohonen, J.; Ilmoniemi, R.J. Modeling of the stimulating field generation in TMS. Electroencephalogr. Clin. Neurophysiol. Suppl. 1999, 51, 30-40.

40. Krieg, S.M.; Lioumis, P.; Makela, J.P.; Wilenius, J.; Karhu, J.; Hannula, H.; Savolainen, P.; Lucas, C.W.; Seidel, K.; Laakso, A.; et al. Protocol for motor and language mapping by navigated TMS in patients and healthy volunteers; workshop report. Acta Neurochir. 2017, 159, 1187-1195. [CrossRef]

41. Lioumis, P.; Zhdanov, A.; Makela, N.; Lehtinen, H.; Wilenius, J.; Neuvonen, T.; Hannula, H.; Deletis, V.; Picht, T.; Makela, J.P. A novel approach for documenting naming errors induced by navigated transcranial magnetic stimulation. J. Neurosci. Methods 2012, 204, 349-354. [CrossRef] [PubMed]

42. Sollmann, N.; Tanigawa, N.; Ringel, F.; Zimmer, C.; Meyer, B.; Krieg, S.M. Language and its right-hemispheric distribution in healthy brains: An investigation by repetitive transcranial magnetic stimulation. NeuroImage 2014, 102 Pt 2, 776-788. [CrossRef] [PubMed]

43. Krieg, S.M.; Sollmann, N.; Tanigawa, N.; Foerschler, A.; Meyer, B.; Ringel, F. Cortical distribution of speech and language errors investigated by visual object naming and navigated transcranial magnetic stimulation. Brain Struct. Funct. 2016, 221, 2259-2286. [CrossRef] [PubMed]

44. Hernandez-Pavon, J.C.; Makela, N.; Lehtinen, H.; Lioumis, P.; Makela, J.P. Effects of navigated TMS on object and action naming. Front. Hum. Neurosci. 2014, 8, 660. [CrossRef]

45. Krieg, S.M.; Tarapore, P.E.; Picht, T.; Tanigawa, N.; Houde, J.; Sollmann, N.; Meyer, B.; Vajkoczy, P.; Berger, M.S.; Ringel, F.; et al. Optimal timing of pulse onset for language mapping with navigated repetitive transcranial magnetic stimulation. NeuroImage 2014, 100, 219-236. [CrossRef]

46. Sollmann, N.; Ille, S.; Negwer, C.; Boeckh-Behrens, T.; Ringel, F.; Meyer, B.; Krieg, S.M. Cortical time course of object naming investigated by repetitive navigated transcranial magnetic stimulation. Brain Imaging Behav. 2016. [CrossRef]

47. Frey, D.; Strack, V.; Wiener, E.; Jussen, D.; Vajkoczy, P.; Picht, T. A new approach for corticospinal tract reconstruction based on navigated transcranial stimulation and standardized fractional anisotropy values. NeuroImage 2012, 62, 1600-1609. [CrossRef]

48. Song, A.W.; Chang, H.C.; Petty, C.; Guidon, A.; Chen, N.K. Improved delineation of short cortical association fibers and gray/white matter boundary using whole-brain three-dimensional diffusion tensor imaging at submillimeter spatial resolution. Brain Connect. 2014, 4, 636-640. [CrossRef]

49. Radbruch, A.; Weberling, L.D.; Kieslich, P.J.; Hepp, J.; Kickingereder, P.; Wick, W.; Schlemmer, H.P.; Bendszus, M. High-Signal Intensity in the Dentate Nucleus and Globus Pallidus on Unenhanced T1-Weighted Images: Evaluation of the Macrocyclic Gadolinium-Based Contrast Agent Gadobutrol. Investig. Radiol. 2015, 50, 805-810. [CrossRef]

50. Krafnick, A.J.; Flowers, D.L.; Napoliello, E.M.; Eden, G.F. Gray matter volume changes following reading intervention in dyslexic children. NeuroImage 2011, 57, 733-741. [CrossRef]

51. Schwarzer, V.; Bahrend, I.; Rosenstock, T.; Dreyer, F.R.; Vajkoczy, P.; Picht, T. Aphasia and cognitive impairment decrease the reliability of rnTMS language mapping. Acta Neurochir. 2018, 160, 343-356. [CrossRef] [PubMed]

52. Duffau, H. Diffusion tensor imaging is a research and educational tool, but not yet a clinical tool. World Neurosurg. 2014, 82, e43-e45. [CrossRef] [PubMed]

53. Conti Nibali, M.; Rossi, M.; Sciortino, T.; Riva, M.; Gay, L.G.; Pessina, F.; Bello, L. Preoperative surgical planning of glioma: Limitations and reliability of fMRI and DTI tractography. J. Neurosurg. Sci. 2019, 63, 127-134. [CrossRef] [PubMed] 
54. Wende, T.; Hoffmann, K.T.; Meixensberger, J. Tractography in Neurosurgery: A Systematic Review of Current Applications. J. Neurol. Surg. A Cent. Eur. Neurosurg. 2020. [CrossRef]

55. Nimsky, C.; Bauer, M.; Carl, B. Merits and Limits of Tractography Techniques for the Uninitiated. Adv. Tech. Stand. Neurosurg. 2016, 37-60. [CrossRef]

(c) (

(C) 2020 by the authors. Licensee MDPI, Basel, Switzerland. This article is an open access article distributed under the terms and conditions of the Creative Commons Attribution (CC BY) license (http://creativecommons.org/licenses/by/4.0/). 